\title{
Corpus Striatum
}

National Cancer Institute

\section{Source}

National Cancer Institute. Corpus Striatum. NCI Thesaurus. Code C12448.

The striped grey and white matter that comprises the caudate and the lentiform nuclei, and is located anterior and lateral to the thalamus in each hemisphere of the brain. 